\title{
The Prevalence of Helicobacter pylori Antibody in Different Age Groups in Central Texas
}

\section{TOM PATTERSON, EMMA STRATEN, STEPHANIE JIMENEZ}

OBJECTIVE: The prevalence of exposure to the bacteria Helicobacter pylori in Central Texas is unknown. It has been shown that elderly individuals have a higher rate of infection than younger individuals. Exposure is even higher in the elderly living in long term care facilities. Evidence of exposure can be demonstrated by the presence of antibody to $H$. pylori. Plasma samples collected from several age groups are tested for the antibody to determine the exposure rate for different age groups.

DESIGN: An exemption was granted by the Texas State University Institutional Review Board (IRB) as the plasma samples had been previously collected for other types of laboratory assessments. Samples were tested with the Status $H$. pylori ${ }^{\oplus}$ Immunoassay that identifies anti-H. pylori IgG antibody in plasma samples.

SETTING: The research study took place in the Texas State University Clinical Laboratory Science Department.

PATIENTS OR OTHER PARTICIPANTS: Blinded in-patient plasma samples were used that had been previously collected for other assays.

MAIN SOURCE MEASURE(S): The percentage of positive antibody tests is determined by age group.

RESULTS: The chi-squared $\left(\mathrm{X}^{2}\right)$ results for each age group expressed a p-value of 0.000 . The age group, 4160 , had the highest rate of positive antibody tests at $24 \%$. The second highest age group was the 61 and over age group at $17 \%$. Third highest age group was the $21-40$ age group at $16 \%$ positive tests. The lowest percentage testing positive was the $0-20$ age group at $6 \%$.

CONCLUSION: There was a higher prevalence of antibody in patients older than 40 years old than in younger patients. Curiously, the oldest age group (61 years or older) did not have the highest rates of exposure. Exposure rates were much lower than rates seen in other areas.

ABBREVIATION: Ethylenediaminetetraacetic acid = EDTA, Statistical Package for Social Sciences ${ }^{\circledast}=$ SPSS $^{\oplus}$, Chi-Squared $=\mathrm{X}^{2}$

INDEX TERMS: Helicobacter pylori, Status H.pylori ${ }^{\oplus}$, duodenal ulcers, peptic (gastric) ulcers, gastric carcinoma

Clin Lab Sci 2012;25(2):102

Tom Patterson, MS, MT (ASCP), Clinical Laboratory Science Program, Texas State University, San Marcos, TX

Emma Straten, Clinical Laboratory Science Program, Texas State University, San Marcos, TX

Stephanie Jimenez, Clinical Laboratory Science Program, Texas State University, San Marcos, TX

Address for Correspondence: Tom Patterson, MS, MT (ASCP), Clinical Laboratory Science Program, Texas State University, 601 University Drive, San Marcos, Texas 78666, 512-245-2791, tp20@txstate.edu

\section{INTRODUCTION}

Helicobacter pylori is a gram-negative, spiral-shaped rod that can colonize in the gastric mucosa. The primary routes of transmission include gastro-oral, fecal-oral and waterborne. ${ }^{1} H$. pylori can cause chronic gastritis and is associated with duodenal and peptic (gastric) ulcers. ${ }^{2} \mathrm{~A}$ large number of individuals that are infected are asymptomatic. In many individuals, histologic and serologic studies have supported the idea that $H$. pylori is a major cause of peptic ulcers. This organism is able to survive in the high acid content of the stomach because it produces large amounts of the enzyme urease. Urease has the ability to hydrolyze urea to form 
ammonia. This creates a micro-environment which neutralizes stomach acid making it possible for the organism to colonize the highly acidic conditions along the gastric mucosa of the stomach. There is strong evidence that long term chronic $H$. pylori infection is associated with active gastritis, peptic ulcers, and gastric malignancies. ${ }^{3}$

Non-invasive tests used for the detection of $H$. pylori include the urea breath test, antibody detection in the plasma, or the H. pylori stool antigen test. ${ }^{4}$ More invasive biopsy tests include culture of the gastric biopsy, urease test in which a portion of the biopsy is incubated in a semisolid agar containing urea, and finally staining of the biopsy material with various stains like hematoxylin and eosin (H\&E), Giemsa, or immunoperoxidase. One study demonstrated that the most sensitive test for the presence of $H$. pylori is immunoperoxidase staining of biopsy material. ${ }^{5}$

A rapid urease test (CLOtest) is also available and can be done at the patient bedside as the biopsy is removed. A portion of biopsy material is placed in small amount of medium containing urea and phenol red. If urease is present in the sample, a color change is immediately apparent. The CLOtest has been found to have lower sensitivity but similar specificity when compared to histology examination. ${ }^{6}$

In some patients, infection with $H$. pylori can begin before the age of five. However, antibody titers in children may not reach adult levels until the age of seven. One study found significantly lower levels of anti- $H$. pylori antibody in young children compared to older children and adults, with prevalence increasing with age. ${ }^{1}$ According to the Annals of Internal Medicine, a study was conducted to determine the prevalence of $H$. pylori (formally know as Campylobacter pylori) in healthy patients as well as patients expressing intestinal illness. Among the healthy population, it was found that $\operatorname{IgG}$ and IgA titers were rarely seen in patients less than 20 years old. However, it was also found that antibody prevalence increased with age. This prevalence reached $50 \%$ in patients that were more than 60 years old. The study also demonstrated high IgG and IgA levels that remained stable for more than one year, which suggests that the organism could persist for at least that period of time. ${ }^{7}$
The investigators of this study chose to utilize the Status H.pylor $i^{\ominus}$ immunoassay as obtaining biopsy materials from a random sample of the population was impractical and unwarranted. The immunoassay detects the presence of $\operatorname{IgG}$ antibody to $H$. pylori in serum, plasma or whole blood. ${ }^{8}$ From the literature review, it was hypothesized that the antibody to $H$. pylori in the population of Central Texas would be higher in patients older than 40 years old than in younger patients. This study is important because the detection of this antibody in different age groups can demonstrate regional exposure rates to this sometimes dangerous organism. Healthcare providers might want to be aware of exposure disparities across age groups in different geographical regions.

\section{MATERIALS AND METHODS}

To investigate the prevalence of the antibody to $H$. pylori in different age groups, plasma samples were used to test for the antibody. Random samples collected in ethylenediaminetetraacetic acid (EDTA) tubes were gathered from a medical center in Central Texas. To detect the antibody in the plasma samples, the Status $H$. pylori $^{\oplus}$ immunoassay was used. The Status $H$. pylori $^{\bullet}$ identifies anti- $H$. pylori IgG antibody in human whole blood, serum, or plasma samples. To help control the variability in specimen conditions, only plasma samples collected in EDTA collection tubes were used. The Status $H$. pylori ${ }^{\oplus}$ test method is an indirect solid-phase immunoassay technology impregnated with the $H$. pylori antigen on the test membrane as well as an $H$. pylori antigen plus anti-human immunoglobulin antibodies coated on gold particles. Ten microliters of the patients' specimen is deposited with a calibrated capillary tube into the upper area of the sample well located below the results window where the reaction takes place. Once the patients' plasma is added, three drops of developer solvent solution are added to mobilize the dye conjugated to $H$. pylori antigen and to the anti-human immunoglobulin antibodies. If antibody is present in the plasma, the dye conjugate binds to the antigen on the membrane, thus a visible band appears in the test area of the membrane. The test result is read and recorded specifically after ten minutes of developer application. To validate that the test method is accurate, the test system provides an internal control. The internal control contains an antibody specific to the $H$. pylori antibody and binds the mobilized antigen in the test. When biopsy/histology is 
used as a reference method, the sensitivity of this test is $95.9 \%$ and the specificity is $89.1 \%{ }^{8}$

Plasma specimens collected from the cold storage room at the medical center were separated into four different age groups. To ensure that there were no privacy violations, the patients' names and other identifiable information was obliterated prior to transport and testing. The specimens were transported from the medical center to the Texas State Clinical Laboratory Science Department and stored at $2^{\circ}$ to $8^{\circ} \mathrm{C}$ prior to antibody testing. The centrifuged plasma EDTA samples were then tested with the Status $H$. pylori ${ }^{\oplus}$ test kit. The four age groups were 0-20 years old, 21-40 years old, 41-60 years old and patients over 61 years of age. Overall, a total of 281 samples was collected, which consisted of 35 samples in the $0-20$ age groups, 96 samples in the 21-40 age groups, 75 samples in the 41 60 age groups, and 75 in the 61 and over age groups. Since the test results collected were nominal (positive or negative) results, the Chi-squared $\left(\mathrm{X}^{2}\right)$ statistical method was used as well as a comparison of the percentage of total positive tests results per age group.

\section{RESULTS}

The data collected from the Status $H$. pylori ${ }^{\oplus}$ test show the percentages of the positive antibody tests (prevalence) as shown in Table 1. Each age group was compared using the Statistical Package for Social Sciences ${ }^{\circledR}$ (SPSS) statistical software for Windows ${ }^{\oplus}$. The chi-squared $\left(\mathrm{X}^{2}\right)$ method was used to assess the data. The data showed significance $(\mathrm{p}=0.00)$ for all age groups. The 0-20 age group (35 samples) showed a $6 \%$ seropositive rate $\left(\mathrm{X}^{2}=27.46\right)$, the $21-40$ age group (96 samples) showed a $16 \%$ seropositive rate $\left(X^{2}=45.36\right)$, the 41-60 age group (75 samples) showed a $24 \%$ seropositive rate $\left(X^{2}=20.28\right)$, while the over 60 age group (75 samples) showed a $17 \%$ seropositive rate $\left(\mathrm{X}^{2}\right.$ $=32.01)$.

Due to specimen availability there were many more specimens available for testing the in the 21-40 age group. To equalize the number of samples across age groups, the youngest age group was not considered for further analysis in Table 2. Due to the differences in sample numbers, the number of samples was reduced to 75 for all remaining age groups. Samples to be eliminated from the data were randomly selected to eliminate any bias. As the samples were tested randomly, the results from the bottom of the randomly tested list were eliminated from the data until the sample number reached 75 . Table 2 shows the percentage of positive antibody tests with the reduction of sample size in the 21-40 age group to 75 . With sample size equalization, the age group with the highest seropositive rate was still the 41-60 age group at 24\% $\left(\mathrm{X}^{2}=20.28\right)$. The 21-40 age group showed a seropositive rate of $12 \%\left(X^{2}=43.32\right)$, while the 61 and over group showed a seropositive rate of $17 \%\left(\mathrm{X}^{2}=\right.$ 32.01). The revised data still showed significance for all age groups $(\mathrm{p}=0.00)$.

Table 1. Percentages of Helicobacter pylori antibody by age group for all samples.

\begin{tabular}{ccccc}
\hline Age Group & Positive & Negative & TOTAL & $\%$ Positive \\
$0-20$ & 2 & 33 & 35 & $6 \%$ \\
$21-40$ & 15 & 81 & 96 & $16 \%$ \\
$41-60$ & 18 & 57 & 75 & $24 \%$ \\
61-over & 13 & 62 & 75 & $17 \%$ \\
TOTAL & 48 & 233 & 281 & \\
\hline
\end{tabular}

Table 2. Percentage of Helicobacter pylori antibody by age group corrected to 75 specimens per group.

\begin{tabular}{ccccc}
\hline Age Group & Positive & Negative & TOTAL & \% Positive \\
$21-40$ & 9 & 66 & 75 & $12 \%$ \\
$41-60$ & 18 & 57 & 75 & $24 \%$ \\
61-over & 13 & 62 & 75 & $17 \%$ \\
TOTAL & 40 & 185 & 225 &
\end{tabular}

\section{DISCUSSION}

The data demonstrated that the antibody to $H$. pylori was more prevalent in the two age groups over 40; with $24 \%$ positive in the $41-60$ group and $17 \%$ positive in the over 60 group. These data suggest that there is a significantly higher prevalence in exposure to this bacteria in individuals over 40 years of age. This finding coincides with other studies that have also found increasing prevalence of the antibody with age. ${ }^{3}$

As previously stated, there were fewer samples in the under 20 age group. This may be due to the fact that the study samples were randomly selected from a bank of previously collected samples of hospitalized patients. The proportion of older hospitalized patients in a nonspecialty medical center is usually much higher than the proportion of younger patients. Most studies have shown that the highest prevalence of $H$. pylori infection 
is in the geriatric population with infection usually by late childhood. ${ }^{3}$ It must be noted that the majority of middle-aged to elderly infected individuals may remain asymptomatic throughout life. ' Surprisingly, the data showed that the over 60 age group had the second highest prevalence. This observation may be due to the more debilitated elderly patients being transferred to long term care or retirement homes or they may be going to specialized hospitals for more serious conditions. The higher positivity rate in the 41-60 age group may be caused by the aforementioned nature of the sample population, the more seriously ill patients in the 61 and over group being cared for in other types of healthcare institutions.

A very high prevalence of exposure to $H$. pylori has been seen in people who have been living in long term care institutions. According to a study conducted by Regev et. al., the prevalence of $H$. pylori among elderly patients ( patients over 70 years of age) admitted to a major medical center in Israel was extremely high and depended on the duration of their stay in a nursing home or long term care facility. Subjects that stayed in these types of facilities for more than 15 months were significantly more likely to be seropositive than subjects with a shorter duration of stay $(84 \%$ and $63 \%$ respectively). This study supported the idea that living in an institution type home with constant contact by healthcare givers and fellow residents is associated with increased risk for $H$. pylori infection in the elderly. ${ }^{10}$ Studies by other investigators seem to demonstrate different levels of exposure in different regions of the world. A study by Pilotto et. al., reported that institutionalized elderly residents in Italy had a seropositive rate of $86 \%$ and a rate of $82 \%$ for the elderly living at home. ${ }^{11}$ It is interesting to note that these data suggest that the exposure rate to H. pylori is much less in Central Texas than exposure rates found in other regions.

Many Americans suffer from gastritis and peptic ulcers. While once thought to be caused by stress, most stomach ulcers are actually caused by the bacteria, Helicobacter pylori. ${ }^{3}$ Worldwide, approximately half of the elderly population has antibodies to $H$. pylori. $^{7}$ In some geographical regions the rate is even higher. Several studies strongly suggest that prolonged $H$. pylori infection of the gastric mucosa may be a significant risk factor for the development of gastritis and gastric carcinoma. For reasons that are unclear, women and blacks that have active infections with $H$. pylori have a higher risk of gastric carcinoma. ${ }^{12}$ With the acknowledgement that this infectious agent can be a cofactor in the cause of several debilitating gastrointestinal diseases, it is important to know what population or subpopulation is at the greatest risk for infection and subsequent morbidity. Studies suggest that elderly residents of nursing homes or other types of institutions may be at even greater risk. More stringent infection control policies and additional staff training might help reduce infection rates among the elderly in these institutions.

\section{ACKNOWLEDGEMENTS}

The investigators would like to recognize and thank: Rodney E. Rohde, PhD, MS, SV, SM/SB(ASCP) ${ }^{\mathrm{CM}}$, Texas State University, Carmen Adams, PhD, Texas State University, Jacella McKenzie, BS (MLS), Texas State University, Stephen A. Garcia, Texas State University, Texas Long Term Care Institute

\section{REFERENCES}

1. Brown LM. Helicobacter pylori: epidemiology and routes of transmission. Epidemiol Rev. 2000;22(2):283-97.

2. Marshall BJ, Warren JR. Unidentified curved bacilli in the stomach of patients with gastritis and peptic ulceration. Lancet. 1984 Jun 16;1(8390):1311-5.

3. Malaty M H. Epidemiology of Helicobacter pylori infection. Best Pract Res Clin Gastroenterol. 2007;21(2):205-14.

4. Tiryaki Z, Yilmaz-Ciftdogan D, Kasigra E. Diagnostic Value of stool antigen and antibody tests for Helicobacter pylori infection in Turkish Children with upper gastrointestinal complaints before and after eradication. Turk J Pediatr. 2010;52:505-11.

5. Loffeld RJ, Stobberingh E, Flendrig JA, Arends JW. Helicobacter pylori in gastric specimens. Comparison of culture, modified giemsa stain, and immunohistochemistry. A retrospective study. J Path. 1991 Sep;165(1):69-73

6. Roma-Giannikou E, Roubani A, Sgouras DN, Panayiotou J, van-Vliet C, Polyzos A, et al. Endoscopic tests for the diagnosis of Helicobacter pylori in children: Validation of rapid urease test. Helicobacter. 2010 Jun;15(3):227-32.

7. Perez - Perez GI, Dworkin BM, Chodos JE, Blaser MJ. Camplylobacter pylori antibodies in humans. Ann Intern Med. 1998;1;109(1):11-7.

8. Princeton BioMeditech Corporation. Status H. Pylori: New One-Step Anti-H. pylori Antibody Test. LifeSign, LLC.

9. Rothenbacher D, Bode G, Peschke F, Berg G, Alder G, Brenner H. Active infection with Helicobacter pylori in an asymptomatic population of middle aged to elderly people. Epidemiol Inf. 1998 Jun;297-303.

10. Regev A, Fraser GM, Braun M, Maoz E, Leibovici L, Niv Y. Seroprevalence of Helicobacter pylori and length of stay in a nursing home. 1999; Helicobacter. 4(2):89-93. 


\section{RESEARCH AND REPORTS}

11. Pilotto A, Fabrello R, Franceschi M, Scagnelli F, Soffiati F, Di Mario F, et al. Helicobacter pylori infection in asymptomatic elderly subjects living at home or in a nursing home: effects on gastric function and nutritional status. Age Ageing 1996;25(3):245-9.
12. Parsonnet J, Friedman GD, Vandersteen DP, Chang Y, Vogelman JH, Orentreich $\mathrm{N}$, et al. Helicobacter pylori infection and risk of gastric carcinoma. $N$ Engl J Med. 1991:325(16):1127-31.

The peer-reviewed Research and Reports Section seeks to publish reports of original research related to the clinical laboratory or one or more subspecialties, as well as information on important clinical laboratory-related topics such as technological, clinical, and experimental advances and innovations. Literature reviews are also included. Direct all inquiries to David $L$ McGlasson MS, MLS, 59th Clinical Research Division/SGRL, 2200Berquist Dr., Bldg. 4430, Lackland AFB TX 782369908, david.mcglasson@lackland.af.mil Clinical Laboratory Science encourages readers to respond with thoughts, questions, or comments regarding these articles. Email responses to westminsterpublishers@comcast.net. In the subject line, please type the journal issue and lead author such as "CLIN LAB SCI 25(2) RE PATTERSON". Selected responses may appear in the Dialogue and Discussion section in a future issue. Responses may be edited for length and clarity. We look forward to hearing from you. 